\title{
Efficacy of peritoneovenous shunt for treating tolvaptan-resistant refractory ascites in a cirrhotic patient with portal vein thrombosis: A case report
}

\author{
KOTA SHIGETO ${ }^{1}$, TAKUMI KAWAGUCHI ${ }^{1}$, TAKASHI NIIZEKI ${ }^{1}$, YASUSHI KUNITAKE ${ }^{1}$, \\ HIDETOSHI TAKEDATSU ${ }^{1}$, TATSUYUKI TONAN ${ }^{2}$, KIMINORI FUJIMOTO ${ }^{2}$, \\ MASATOSHI TANAKA ${ }^{3}$, TOSHI ABE ${ }^{2}$, HISANORI NAITO ${ }^{3}$ and TAKUJI TORIMURA ${ }^{1}$ \\ ${ }^{1}$ Department of Medicine, Division of Gastroenterology; ${ }^{2}$ Department of Radiology, \\ Kurume University School of Medicine, Kurume, Fukuoka 830-0011; ${ }^{3}$ Naito Hospital, Kurume, Fukuoka 830-0038, Japan
}

Received January 15, 2015; Accepted February 8, 2016

DOI: $10.3892 / 01.2016 .4357$

\begin{abstract}
Peritoneovenous shunt is normally used for the treatment of refractory ascites. However, its efficacy in treating tolvaptan-resistant refractory ascites has not been reported thus far. In addition, the impact of peritoneovenous shunt on the prognosis of cirrhotic patients remains controversial. In the present report, a case of tolvaptan-resistant refractory ascites associated with liver cirrhosis and portal vein thrombosis is described. The male patient was diagnosed with hepatitis $\mathrm{C}$ virus-related liver cirrhosis at the age of 51 years. At the age of 56 years, the patient developed portal vein thrombosis, resulting in the development of refractory ascites. Since the ascites was resistant to treatment with a low-sodium diet and diuretics such as tolvaptan, a peritoneovenous shunt was implanted upon obtaining consent. The shunt immediately increased the urine volume, and the ascites was markedly decreased. The patient's body weight decreased from 62.7 to $57.1 \mathrm{~kg}$ in 2 days, and his ascites symptom inventory-7 score decreased from 23 to 0 points in 31 days. Although the patient succumbed to sepsis on day 486 following the shunt implant, his activities of daily living were preserved until 8 days prior to mortality. Thus, the present case supports the efficacy of peritoneovenous shunt for the treatment of tolvaptan-resistant refractory ascites associated with liver cirrhosis and portal vein thrombosis. Furthermore, the present case suggests that peritoneovenous shunt may prolong the survival of cirrhotic patents with refractory ascites.
\end{abstract}

Correspondence to: Dr Takumi Kawaguchi, Department of Medicine, Division of Gastroenterology, Kurume University School of Medicine, 67 Asahi-Machi, Kurume, Fukuoka 830-0011, Japan

E-mail: takumi@med.kurume-u.ac.jp

Abbreviations: ADL, activities of daily living; ASI-7, ascites symptom inventory-7

Key words: peritoneovenous shunt, refractory ascites, prognosis, quality of life, activities of daily living

\section{Introduction}

Ascites that does not resolve with standard medical treatment such a low-sodium diet and diuretics is known as refractory ascites, and is frequently associated with the development of hepatorenal syndrome, spontaneous bacterial peritonitis and dilutional hyponatremia (1). Refractory ascites also causes a loss of appetite and muscle wasting, and impairs the activities of daily living (ADL) (1). Thus, refractory ascites is a life-threatening complication that lowers the quality of life of cirrhotic patients, and is an independent predictor of short survival $(1,2)$.

One of the main reasons for cirrhosis-related water retention is a reduced ability of the kidneys to excrete electrolyte-free water, due to an increase in the levels of arginine vasopressin (3). Arginine vasopressin receptor antagonists, a novel class of diuretics, have been recently approved for the treatment of cirrhosis-related fluid retention in Japan $(3,4)$. These diuretics antagonize vasopressin V2 receptors, resulting in the inhibition of electrolyte-free water reabsorption and an increase in electrolyte-free water excretion (5). Tolvaptan, a vasopressin V2 receptor antagonist, improves hepatic edema and reduces ascites in cirrhotic patients $(6,7)$. However, tolvaptan may not always be effective for treating refractory ascites, since there are various mechanisms involved in the development of this condition $(1,8)$.

Peritoneovenous shunt was designed to transport ascites from the peritoneal cavity back into the central venous circulation, and is used for the treatment of refractory ascites $(9,10)$. Peritoneovenous shunt is effective in relieving refractory ascites, and it decreases the required dose of diuretics and the duration and number of hospitalizations, compared with paracentesis with intravenous infusion of albumin (11). However, the efficacy of peritoneovenous shunt on tolvaptan-resistant refractory ascites has not been reported to date. In addition, the impact of peritoneovenous shunt on the prognosis of cirrhotic patients with refractory ascites remains controversial, due to the severe potential complications of peritoneovenous shunt, including disseminated intravascular coagulation (11-13).

In the present study, a case of tolvaptan-resistant refractory ascites associated with liver cirrhosis and portal vein thrombosis is described. Peritoneovenous shunt markedly 
reduced the ascites and improved the ADL score of the patient. Although the patient succumbed to sepsis at 486 days following implantation of the shunt, the ascites was under control. Thus, the present case indicates that peritoneovenous shunt relieves tolvaptan-resistant ascites, improves ADL and prolongs survival in cirrhotic patients.

\section{Case report}

In June 2014, a 51-year-old Japanese man was referred to the Kurume University Hospital, affiliated with Kurume University School of Medicine (Kurume, Japan), for examination of persistent hepatic dysfunction. The patient was diagnosed with hepatitis C virus-related liver cirrhosis based on serological findings (Table I). Although the patient was treated with peginterferon (Pegintron ${ }^{\circledR} ; 60 \mu \mathrm{g}$ once a week; MSD K.K., Tokyo, Japan) and ribavirin (Rebetol ${ }^{\circledR} ; 600 \mathrm{mg} /$ day; MSD K.K.) combination therapy for 48 weeks, the hepatitis C virus was not eradicated. Therefore, the patient was treated with ursodeoxycholic acid (Urso ${ }^{\circledR} ; 600 \mathrm{mg}$ /day; Mitsubishi Tanabe Pharma Corporation, Tokyo, Japan) and a glycyrrhizin-containing preparation (Stronger neo-minophagen $\mathbf{C}^{\circledR}$; $40 \mathrm{ml}$ twice a week; Minophagen Pharmaceutical Co., Ltd., Tokyo, Japan) for 5 years.

At the age of 56 years, the patient developed an infected urachal cyst that was resistant to antibiotic medication. Following surgical resection of the infected urachal cyst, the patient developed portal vein thrombosis. Although the patient was treated with anticoagulant therapy using warfarin potassium (Warfarin ${ }^{\circledR} ; 1.5 \mathrm{mg} /$ day; Eisai Co. Ltd., Tokyo, Japan) for 1 year, the portal vein thrombosis did not improve. Massive thrombosis developed at the umbilical portion of the portal vein, as confirmed by magnetic resonance imaging (Signa HDxt1.5T ${ }^{\circledR}$; GE Healthcare Japan, Tokyo, Japan) using gadolinium ethoxybenzyl diethylenetriaminepentaacetic acid (EOB Primovist ${ }^{\circledR}$; Bayer, Osaka, Japan) (Fig. 1A). In addition, the patient accumulated a large amount of ascitic fluid (Fig. 1B).

The biochemical parameters on admission indicated decompensated liver cirrhosis (Table I), in addition to the following ascitic findings: Yellow-tinged appearance; serum-ascites albumin gradient of 2.39; fluid-serum total protein ratio of 0.163 ; $\alpha$-fetoprotein levels of $0.6 \mathrm{ng} / \mathrm{ml}$; carcinoembryonic antigen levels of $0.5 \mathrm{ng} / \mathrm{ml}$; carbohydrate antigen 19-9 levels of $6.8 \mathrm{U} / \mathrm{ml}$; and cell number of neutrophils 35 cells $/ \mu 1$, suggesting transudate ascites. The patient was treated with a low-sodium diet (salt, $7 \mathrm{~g} /$ day), diuretics [furosemide (Lasix ${ }^{\circledR}$; Sanofi K.K., Tokyo, Japan), $60 \mathrm{mg} / \mathrm{day}$, and spironolactone (Aldactone- $\mathrm{A}^{\circledR}$; Pfizer Japan Inc., Tokyo, Japan), $100 \mathrm{mg} /$ day] for 1 year and an albumin preparation (Albuminar ${ }^{\circledR} ; 12.5 \mathrm{~g}$, three times a month; CSL Behring K.K., Tokyo, Japan) for 4 months; however, the amount of ascitic fluid was not reduced (Fig. 2). Tolvaptan (Samusuca $^{\circledR}$; 7.5 mg/day; Otsuka Pharmaceutical Co., Ltd., Tokyo, Japan) was added to the treatment on day 16 , and was continued for 4 months, but the ascites remained resistant to treatment (Fig. 2). Thus, the refractory ascites did not improve by diet or medication, including tolvaptan, and the patient gradually lost appetite.

The patient presented a Child-Pugh score of 12 points (Table I). In consequence, the patient was informed about his predicted poor prognosis, and was communicated that liver
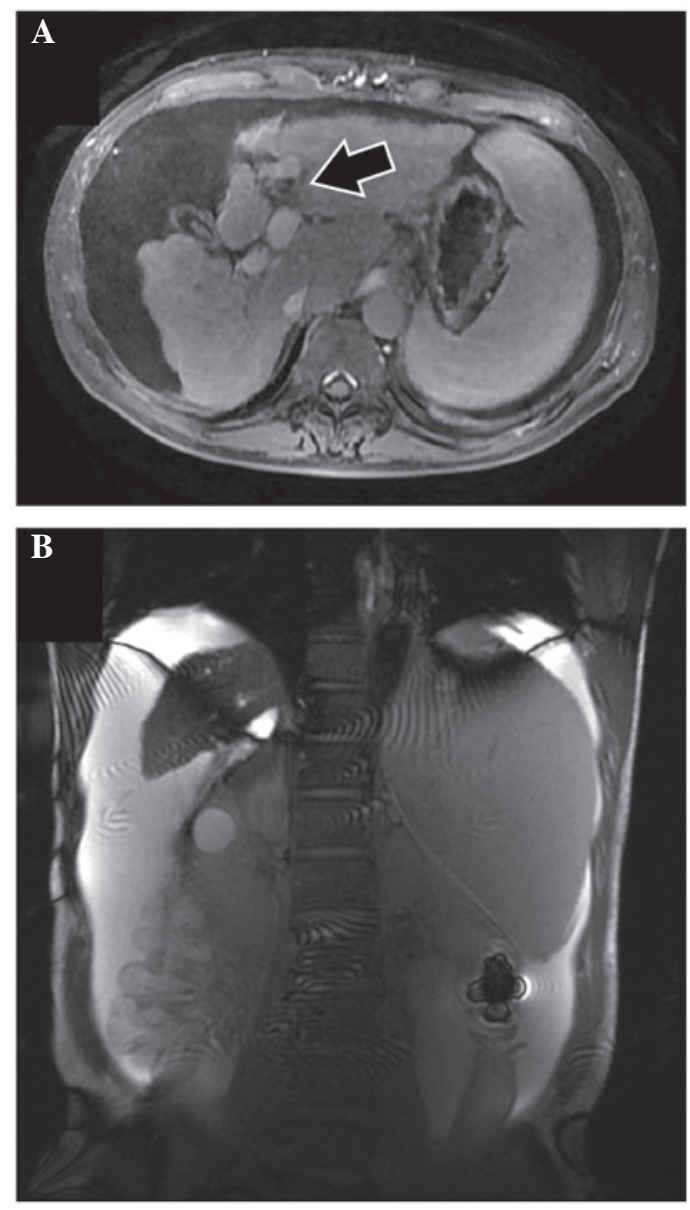

Figure 1. Abdominal magnetic resonance imaging. (A) In the portal phase of a dynamic study using gadolinium ethoxybenzyl diethylenetriaminepentaacetic acid, massive thrombosis is observed at the umbilical portion of the portal vein (arrow). (B) A large amount of ascitic fluid is observed on T2-weighted imaging.

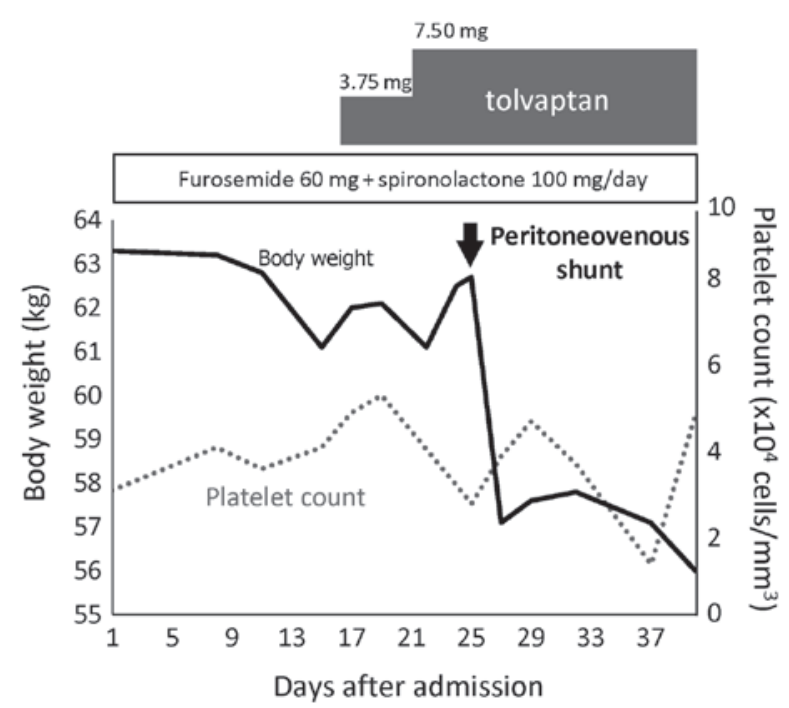

Figure 2. Course of body weight and platelet count following treatment with diuretics and implantation of a peritoneovenous shunt.

transplantation was the only possible therapeutic strategy associated with long-term survival. However, the patient did not consent to liver transplantation; therefore, a peritoneovenous 
Table I. Biochemical parameters of the patient on admission.

\begin{tabular}{|c|c|c|}
\hline Parameters & Value & Reference value \\
\hline Red blood cell count $\left(\times 10^{4}\right.$ cells $\left./ \mathrm{mm}^{3}\right)$ & 346.00 & $380.00-500.00$ \\
\hline Hemoglobin levels (g/dl) & 11.30 & $11.00-15.00$ \\
\hline White blood cell count (cells $/ \mathrm{mm}^{3}$ ) & $4,100.00$ & $4,000.00-9,000.00$ \\
\hline Platelet count $\left(\times 10^{4}\right.$ cells $\left./ \mathrm{mm}^{3}\right)$ & 3.10 & $13.00-36.00$ \\
\hline Aspartate transaminase levels (U/l) & 49.00 & $13.00-33.00$ \\
\hline Alanine aminotransferase levels (U/l) & 23.00 & $8.00-42.00$ \\
\hline Lactate dehydrogenase levels (U/l) & 303.00 & $119.00-229.00$ \\
\hline Alkaline phosphatase levels (U/l) & 202.00 & $115.00-359.00$ \\
\hline$\gamma$-glutamyltranspeptidase levels (U/1) & 14.00 & $10.00-47.00$ \\
\hline Total protein levels (g/dl) & 6.61 & $6.70-8.30$ \\
\hline Albumin levels (g/dl) & 2.96 & $4.00-5.00$ \\
\hline Total bilirubin levels (mg/dl) & 2.54 & $0.30-1.50$ \\
\hline C-reactive protein levels (mg/dl) & 1.39 & $<0.40$ \\
\hline Total cholesterol levels (mg/dl) & 125.00 & $128.00-220.00$ \\
\hline Fasting blood glucose levels (mg/dl) & 114.00 & $80.00-109.00$ \\
\hline Hemoglobin A1c levels (\%) & 4.50 & $4.30-5.80$ \\
\hline Prothrombin activity (\%) & 31.00 & $60.00-130.00$ \\
\hline Blood urea nitrogen levels (mg/dl) & 13.80 & $8.00-22.00$ \\
\hline Creatinine levels (mg/dl) & 0.69 & $0.40-0.70$ \\
\hline Blood ammonia levels $(\mu \mathrm{g} / \mathrm{dl})$ & 69.00 & $12.00-66.00$ \\
\hline$\alpha$-fetoprotein levels (ng/ml) & 1.90 & $<8.70$ \\
\hline Carcinoembryonic antigen levels (ng/ml) & 4.20 & $<5.00$ \\
\hline Carbohydrate antigen 19-9 levels (U/ml) & 32.20 & $<37.00$ \\
\hline Child-Pugh score & 12.00 & $\mathrm{~N} / \mathrm{A}$ \\
\hline MELD score ${ }^{\mathrm{a}}$ & 18.00 & $\mathrm{~N} / \mathrm{A}$ \\
\hline MELD-Na score ${ }^{a}$ & 23.00 & N/A \\
\hline
\end{tabular}

${ }^{a}$ MELD and MELD-Na scores were calculated using the Mayo Clinic website (http://www.mayoclinic.org/medical-professionals/model-endstage-liver-disease/meld-na-model). MELD, model for end-stage liver disease; Na, sodium; N/A, not applicable.

Table II. Body weight and biochemical parameters prior to and on day 456 following implantation of the peritoneovenous shunt.

\begin{tabular}{|c|c|c|}
\hline Characteristics & Prior to shunt & 456 days following shunt \\
\hline Body weight (kg) & 62.70 & 58.90 \\
\hline Platelet count $\left(\mathrm{x} 10^{4}\right.$ cells $\left./ \mathrm{mm}^{3}\right)$ & 3.10 & 2.20 \\
\hline Aspartate transaminase levels (U/l) & 49.00 & 39.00 \\
\hline Alanine aminotransferase levels (U/l) & 23.00 & 26.00 \\
\hline$\gamma$-glutamyltranspeptidase levels (U/l) & 14.00 & 14.00 \\
\hline Total bilirubin levels (mg/dl) & 2.54 & 3.56 \\
\hline Albumin levels (g/dl) & 2.96 & 2.43 \\
\hline Prothrombin activity $(\%)$ & 34.00 & 59.00 \\
\hline Cholinesterase levels (U/l) & 49.00 & 48.00 \\
\hline Creatinine levels (mg/dl) & 0.69 & 0.48 \\
\hline Sodium levels (mEq/l) & 131.00 & 133.00 \\
\hline Child-Pugh score & 12.00 & 12.00 \\
\hline MELD score ${ }^{\mathrm{a}}$ & 18.00 & 14.00 \\
\hline MELD-Na score ${ }^{a}$ & 23.00 & 23.00 \\
\hline
\end{tabular}

${ }^{a}$ MELD and MELD-Na scores were calculated using the Mayo Clinic website (http://www.mayoclinic.org/medical-professionals/model-endstage-liver-disease/meld-na-model). MELD, model for end-stage liver disease; Na, sodium. 


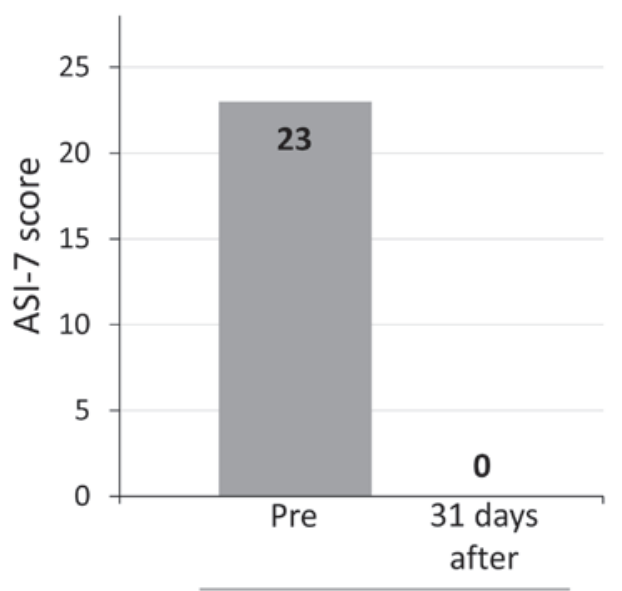

Treatment

Figure 3. Ascites symptom inventory-7 score prior to and on day 31 following implantation of the peritoneovenous shunt. ASI-7, ascites symptom inventory-7; Pre, pretreatment.

shunt was implanted upon obtaining consent to relieve the ascites-associated symptoms on day 25 . Following implantation of the shunt, the volume of urine immediately increased and the ascites markedly decreased. The patient's body weight decreased from 62.7 to $57.1 \mathrm{~kg}$ in 2 days (Fig. 2). Although the patient exhibited disseminated intravascular coagulation on day 37, this condition improved with the use of thrombomodulin alfa (Recomoduin ${ }^{\circledR} ; 265,000$ U/day; Pfizer Japan Inc.) for 3 days and antibiotic treatment [piperacillin (Pentcillin ${ }^{\circledR}$; 2 g/day for 7 days; Taisho Toyama Pharmaceutical Co., Ltd., Tokyo, Japan) and levofloxacin (Cravit; 500 mg/day for 10 days; Daiichi Sankyo Company, Ltd., Tokyo, Japan)]. The patient's ascites symptom inventory-7 (ASI-7) score, an ascites-specific symptom scale (14), decreased from 23 to 0 points on day 56 (Fig. 3). Along with the reduction of the ascites, the patient's appetite improved, and therefore the patient was discharged from the hospital.

A computed tomography scan (Discovery CT750 HD ${ }^{\circledR}$; GE Healthcare Japan) revealed that the patient's ascites remained well controlled at $>450$ days following implantation of the shunt, and his liver function did not worsen, despite lowering the doses of diuretics administered to the patient (furosemide, $20 \mathrm{mg} /$ day and spironolactone, $50 \mathrm{mg} /$ day) and withdrawing tolvaptan (Fig. 4; Table II). The patient developed infectious diseases, including pulmonary cryptococcosis, and short-term admission was required in four occasions during the 458-day period subsequent to the implantation of the shunt. Despite the infections, the patient experienced a normal daily life with his family. The patient succumbed to sepsis, probably due to bacterial translocation from the intestine, on day 486 following implantation of the shunt. However, his ADL was preserved until 8 days prior to mortality.

\section{Discussion}

In the present report, a case of tolvaptan-resistant refractory ascites associated with liver cirrhosis and portal vein thrombosis is described. Peritoneovenous shunt markedly reduced the ascites and ASI-7 score. Although the patient experienced

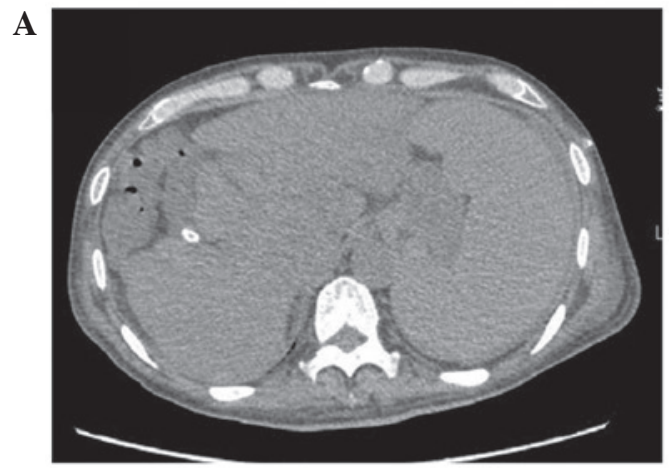

B

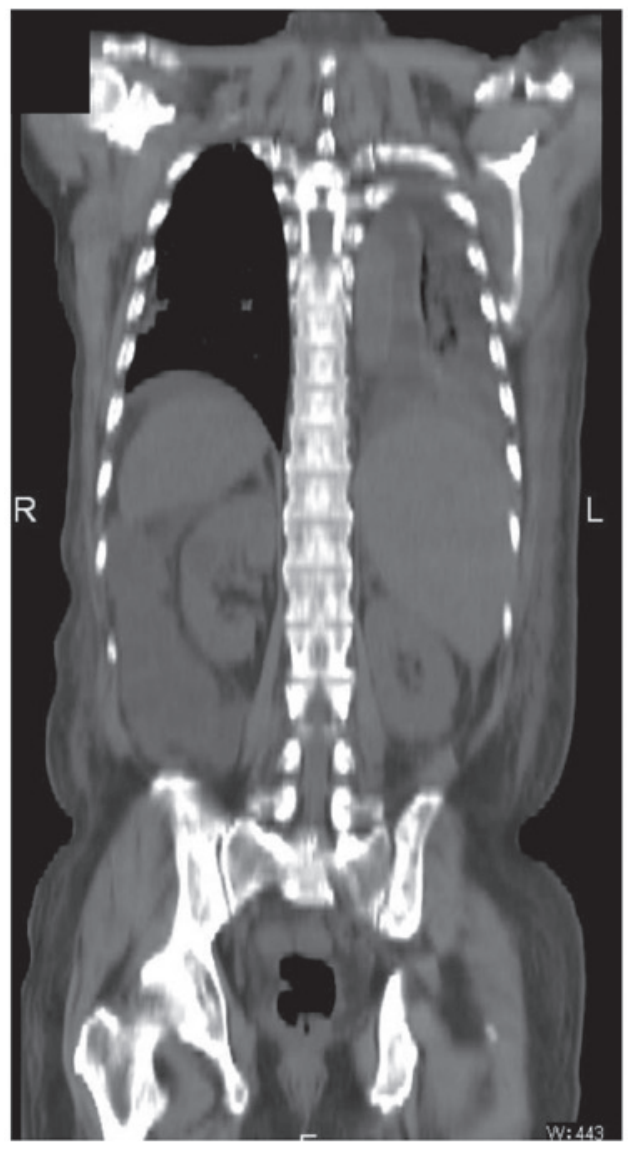

Figure 4. Abdominal computed tomographic imaging performed at 437 days following the implantation of a peritoneovenous shunt. No marked ascites is observed in the (A) transected or (B) coronal images. R, right; L, left.

recurrent infectious diseases and succumbed to sepsis on day 486 following implantation of the shunt, the ascites was under control. Thus, the present case supports the efficacy of peritoneovenous shunt in treating tolvaptan-resistant refractory ascites in cirrhotic patients with portal vein thrombosis. In addition, peritoneovenous shunt may prolong the survival of cirrhotic patents with refractory ascites.

The refractory ascites in the present patient was initially treated with tolvaptan in combination with conventional diuretic therapy. However, the patient did not respond to this treatment. At 31 days following the implantation of the peritoneovenous shunt, the tolvaptan-resistant refractory ascites was markedly reduced, and the patient's symptoms, as evaluated by ASI-7 score, disappeared. Although it is unclear why his ascites did not respond to tolvaptan treatment, a possible reason is that the ascites was not caused by a reduced ability 
to excrete solute-free-water through an increase in arginine vasopressin levels, which is the most common cause of cirrhosis-related water retention (3). In the present case, the ascites became intractable following the development of portal vein thrombosis; therefore, severe portal hypertension appears to be the main pathogenesis of the refractory ascites exhibited by the patient. Thus, etiological differences in refractory ascites may account for a lack of response to tolvaptan treatment. To the best of our knowledge, the present case report is the first to demonstrate the efficacy of peritoneovenous shunt for the treatment of tolvaptan-resistant refractory ascites in a cirrhotic patient with portal vein thrombosis.

Although peritoneovenous shunt is known to be effective in relieving refractory ascites, Nitta et al (12) reported that peritoneovenous shunt should not be considered in cirrhotic patients with refractory ascites and prolonged prothrombin time, due to an increase in the risk of disseminated intravascular coagulation. Ginès et al (11) performed a randomized controlled trial and demonstrated that peritoneovenous shunt did not prolong the survival of cirrhotic patients with refractory ascites, compared with patients treated with large-volume paracentesis plus intravenous albumin. In the present case, the patient exhibited hepatitis $C$ virus-related decompensated liver cirrhosis with portal vein thrombosis, and his Child-Pugh score was 12 points. Although it is unclear whether peritoneovenous shunt improved the prognosis, Heuman et al (15) reported a 180-day survival rate of $58.6 \%$ in cirrhotic patients with persistent ascites and low levels of sodium in serum. Girleanu et al (16) examined the natural course of nonmalignant partial portal vein thrombosis in cirrhotic patients, and reported a 6-month survival rate of $66.66 \%$ and a median survival time of 8.6 months in patients with worsening portal vein thrombosis. These findings support a prolonged survival with implantation of peritoneovenous shunt in the present patient. In addition, Miyaaki et al (13) reported two patients who experienced an increase in liver volume following implantation of a peritoneovenous shunt, further supporting the possibility that survival is prolonged with peritoneovenous shunt.

The ASI-7 score of the present patient markedly improved with the disappearance of the refractory ascites following implantation of the shunt. However, the patient experienced recurrent infectious diseases and succumbed to sepsis on day 486 following implantation of the shunt. Recent advances in the treatment of malnourishment, ascites, esophageal varices and hepatocellular carcinoma indicate that infectious diseases may be one of the major causes of mortality in patients with liver cirrhosis in the future (17,18). Arvaniti et al (19) reported that infections increased mortality by 4 -fold in cirrhotic patients, and that $30 \%$ of cirrhotic patients succumbed to disease within 1 month following the infection, and an additional 30\% succumbed to disease within 1 year. Therefore, further studies in patients with advanced liver cirrhosis should be focused on the management of infectious diseases.

In conclusion, the present study describes a case of tolvaptan-resistant refractory ascites related to liver cirrhosis with portal vein thrombosis. The patient was subjected to peritoneovenous shunt, which markedly reduced the ascites and the ASI-7 score. Although the patient succumbed to sepsis on day 486 subsequent to the implantation of the shunt, his ascites was under control. Thus, the present case indicates that peritoneovenous shunt relieves tolvaptan-resistant ascites and improves ADL. In addition, the findings of the present case indicate that peritoneovenous shunt may prolong the survival of cirrhotic patents with refractory ascites.

\section{References}

1. Salerno F, Guevara M, Bernardi M, Moreau R, Wong F, Angeli P, Garcia-Tsao G and Lee SS: Refractory ascites: Pathogenesis, definition and therapy of a severe complication in patients with cirrhosis. Liver Int 30: 937-947, 2010.

2. Arroyo V, Ginès P, Gerbes AL, Dudley FJ, Gentilini P, Laffi G, Reynolds TB, Ring-Larsen H and Schölmerich J: Definition and diagnostic criteria of refractory ascites and hepatorenal syndrome in cirrhosis. International Ascites Club. Hepatology 23: 164-176, 1996.

3. Boyer TD: Tolvaptan and hyponatremia in a patient with cirrhosis. Hepatology 51: 699-702, 2010.

4. Okita K, Kawazoe S, Hasebe C, Kajimura K, Kaneko A, Okada M and Sakaida I; ASCITES Dose-Finding Trial Group: Dose-finding trial of tolvaptan in liver cirrhosis patients with hepatic edema: A randomized, double-blind, placebo-controlled trial. Hepatol Res 44: 83-91, 2014.

5. Schrier RW, Gross P, Gheorghiade M, Berl T, Verbalis JG, Czerwiec FS and Orlandi C; SALT Investigators: Tolvaptan, a selective oral vasopressin V2-receptor antagonist, for hyponatremia. N Engl J Med 355: 2099-2112, 2006.

6. Okita K, Sakaida I, Okada M, Kaneko A, Chayama K, Kato M, Sata M, Yoshihara H, Ono N and Murawaki Y: A multicenter, open-label, dose-ranging study to exploratively evaluate the efficacy, safety and dose-response of tolvaptan in patients with decompensated liver cirrhosis. J Gastroenterol 45: 979-987, 2010.

7. Sakaida I, Kawazoe S, Kajimura K, Saito T, Okuse C, Takaguchi K, Okada M and Okita K; ASCITES-DOUBLEBLIND Study Group: Tolvaptan for improvement of hepatic edema: A phase 3 , multicenter, randomized, double-blind, placebo-controlled trial. Hepatol Res 44: 73-82, 2014.

8. Dahl E, Gluud LL, Kimer N and Krag A: Meta-analysis: The safety and efficacy of vaptans (tolvaptan, satavaptan and lixivaptan) in cirrhosis with ascites or hyponatraemia. Aliment Pharmacol Ther 36: 619-626, 2012.

9. Leveen HH, Christoudias G, Ip M, Luft R, Falk G and Grosberg S: Peritoneo-venous shunting for ascites. Ann Surg 180: 580-591, 1974.

10. Martin LG: Percutaneous placement and management of peritoneovenous shunts. Semin Intervent Radiol 29: 129-134, 2012.

11. Ginès $\mathrm{P}$, Arroyo V, Vargas V, et al: Paracentesis with intravenous infusion of albumin as compared with peritoneovenous shunting in cirrhosis with refractory ascites. N Engl J Med 325: 829-835, 1991.

12. Nitta H, Okamura S, Mizumoto T, et al: Prognosis assessment of patients with refractory ascites treated with a peritoneovenous shunt. Hepatogastroenterology 60: 1607-1610, 2013.

13. Miyaaki H, Murakami E, Ichikawa T, et al: Long-term increase in liver volume after Denver peritoneovenous shunt: Report of two cases. Liver Int 29: 774-775, 2009

14. Onishi Y, Wakita T, Fukuhara S, et al: Development and validation of a symptom scale specific for ascites accompanied with cirrhosis: The ASI-7. Clin Transl Gastroenterol 5: e48, 2014.

15. Heuman DM, Abou-Assi SG, Habib A, Williams LM, Stravitz RT, Sanyal AJ, Fisher RA and Mihas AA: Persistent ascites and low serum sodium identify patients with cirrhosis and low MELD scores who are at high risk for early death. Hepatology 40: 802-810, 2004

16. Girleanu I, Stanciu C, Cojocariu C, et al: Natural course of nonmalignant partial portal vein thrombosis in cirrhotic patients. Saudi J Gastroenterol 20: 288-292, 2014.

17. Kawaguchi T, Shiraishi K, Ito T, et al: Branched-chain amino acids prevent hepatocarcinogenesis and prolong survival of patients with cirrhosis. Clin Gastroenterol Hepatol 12: 1012-1018. e1, 2014.

18. Nahon $\mathrm{P}$, Lescat $\mathrm{M}$, Layese $\mathrm{R}$, et al: Bacterial infection in compensated viral cirrhosis impairs 5-year survival (ANRS CO12 CirVir prospective cohort). Gut: gutjnl-2015-310275, 2015 (Epub ahead of print).

19. Arvaniti V, D'Ámico G, Fede G, Manousou P, Tsochatzis E, Pleguezuelo $M$ and Burroughs AK: Infections in patients with cirrhosis increase mortality four-fold and should be used in determining prognosis. Gastroenterology 139: 1246-1256, 2010. 